8th Alexander Friedmann International Seminar

on Gravitation and Cosmology

International Journal of Modern Physics: Conference Series

Vol. 3 (2011) 303-311

(C) World Scientific Publishing Company

DOI: $10.1142 /$ S2010194511001401

\title{
A STRAINED SPACE-TIME TO EXPLAIN THE LARGE SCALE PROPERTIES OF THE UNIVERSE
}

\author{
ANGELO TARTAGLIA \\ DIFIS, Politecnico di Torino, Corso Duca degli Abruzzi 24 \\ Torino, 10129, Italy \\ and \\ INFN, Via P. Giuria 1, Torino, 10126, Italy \\ angelo.tartaglia@polito.it
}

Received 3 June 2011

\begin{abstract}
Space-time can be treated as a four-dimensional material continuum. The corresponding generally curved manifold can be thought of as having been obtained, by continuous deformation, from a flat four-dimensional Euclidean manifold. In a three-dimensional ordinary situation such a deformation process would lead to strain in the manifold. Strain in turn may be read as half the difference between the actual metric tensor and the Euclidean metric tensor of the initial unstrained manifold. On the other side we know that an ordinary material would react to the attempt to introduce strain giving rise to internal stresses and one would have correspondingly a deformation energy term. Assuming the conditions of linear elasticity hold, the deformation energy is easily written in terms of the strain tensor. The Einstein-Hilbert action is generalized to include the new deformation energy term. The new action for space-time has been applied to a Friedmann-LemaitreRobertson-Walker universe filled with dust and radiation. The accelerated expansion is recovered, then the theory has been put through four cosmological tests: primordial isotopic abundances from Big Bang Nucleosynthesis; Acoustic Scale of the CMB; Large Scale Structure formation; luminosity/redshift relation for type Ia supernovae. The result is satisfying and has allowed to evaluate the parameters of the theory.
\end{abstract}

Keywords: Cosmology; space-time; elasticity.

PACS numbers: 98.80.-k, 95.36.+x, 95.30.Sf

\section{Introduction}

Space and time are fundamental concepts for physics, but their nature has always been neither well understood nor well defined. General Relativity (GR) provides a paradigm in which space and time are tied together in a four-dimensional Riemannian manifold with Lorentzian signature. Such a manifold is endowed with geometrical properties and its curvature is assumed to depend on the presence of matter/energy, which is something else with respect to space-time. All this has led to the world famous Einstein equations:

$$
G_{\mu \nu}=\kappa T_{\mu \nu}
$$


Often, however, one has the sensation that space-time is treated mostly as a smart mathematical trick in order to have the physics working and nothing else, even though Eq. (1) tell us that the left hand side lives on its own and interacts with the right hand side which represents matter/energy. It is also true that the tensorial properties we use to describe the behaviour of space-time are applied as well, in three dimensions, to material continua and the theory of elasticity.

Moving from the above remarks I present here a theory of space-time where the four-dimensional manifold we use as a scenario for GR is treated as a real continuum endowed with specific elastic properties. This idea is not new, but for a long time it has been considered as being purely formal; only from the 60's of the last century people have started to consider the possibility that the macroscopic properties of space-time could emerge out of some microscopic quantum structure, more or less as it happens with the classical elasticity theory in three dimensions. ${ }^{1-5}$ The idea that the geometrical description of gravity is an emergent manifestation of some quantum microscopic structure underlies also Padmanabhan's interpretation of the action integral of space-time as an entropy functional. ${ }^{6,7}$

The present author, together with his collaborators, has studied the purely classical aspects of this approach, developing step by step ${ }^{8-11}$ the basics of the theory which is expounded here: the Strained State Theory (SST). The present paper will add the use of an Euclidean manifold (rather than Minkowskian) as the start and will present and comment four cosmic tests. The two ideas from which the theory is developed are: a) the actual space-time is obtained by continuous deformation from an Euclidean flat four-dimensional manifold; b) the deformation implies the presence of a potential term which must be included in the action integral. From these assumptions the behavior of a Friedmann-Lemaittre-Robertson-Walker (FLRW) has been obtained. The result has been checked using four typical cosmological tests: the relative abundance of the isotopes produced in the primordial nucleosynthesis (Big Bang Nucleosynthesis, BBN); the Acoustic Scale of the CMB; the Large Scale Structure formation (LSS), and the luminosity/redshift curve of type Ia supernovae (SnIa).

As we shall see, all tests are reasonably satisfying, thus showing that the SST is a good framework within which to describe the large scale phenomena of the universe. In the following the fundament of the theory is presented, then the application to a homogeneous and isotropic universe; next the cosmological tests will be discussed, and finally perspectives and open problems will be presented.

\section{The Strained Space-Time}

As said in the Introduction, let us consider an Euclidean four-dimensional space and let us proceed to a continuous deformation of it until a final curved manifold is obtained. This procedure is easily described in a higher dimensional flat embedding where both manifolds are shown: the so called 'reference manifold' (the Initial Euclidean one) and the named 'natural manifold' (corresponding to the actual 


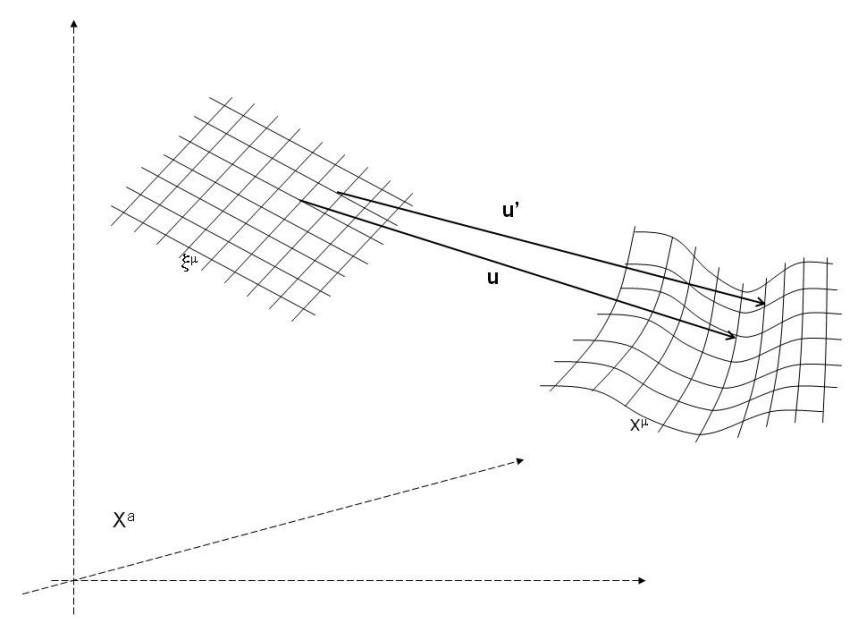

Fig. 1. The embedding $(N+n)$-dimensional space is shown with the two embedded manifolds. $X^{a}$ 's are the coordinates of the embedding; $a$ runs from 1 to $N+n$. The $\xi^{\mu}$ 's and the $x^{\mu}$ 's are the coordinates on the reference and natural manifolds; $\mu$ runs from 1 to $N$. The $\mathbf{u}$ and $\mathbf{u}^{\prime}$ vectors represent the vector displacement field.

space-time we wish to describe). ${ }^{12}$ The flatness of the embedding manifold is important because it allows the definition of a global displacement vector field $\mathbf{u}$ establishing a one-to-one correspondence between pairs of positions on the two lower dimensional manifolds. The number of extra-dimensions needed will in general be 1 , but it does actually depend on the features of the natural manifold: if the latter has peculiar symmetries and, above all, contains any singularity the additional dimensions needed to insure a flat embedding can be more than 1 . The situation is pictorially presented in Fig. 1.

Comparing corresponding line elements on the two $N$-dimensional manifolds and using the Euclidean metric tensor, $E_{\mu \nu}$, in one case, and the natural metric tensor, $g_{\mu \nu}$, in the other, we see that it is:

$$
g_{\mu \nu}=E_{\mu \nu}+2 \varepsilon_{\mu \nu}
$$

with $^{10}$

$$
\varepsilon_{\mu \nu}=\frac{1}{2}\left(E_{a \mu} \frac{\partial u^{a}}{\partial x^{\nu}}+E_{\nu b} \frac{\partial u^{b}}{\partial x^{\mu}}+E_{a b} \frac{\partial u^{a}}{\partial x^{\mu}} \frac{\partial u^{b}}{\partial x^{\nu}}\right) .
$$

The tensor $\varepsilon_{\mu \nu}$ is the strain tensor, in full analogy with the three-dimensional situation in a continuous material subject to deformation originating from any cause.

\subsection{Defects}

Pursuing the analogy between an $N$-dimensional continuum and three-dimensional continua we may introduce the concept of defect. In the classical definition given by 


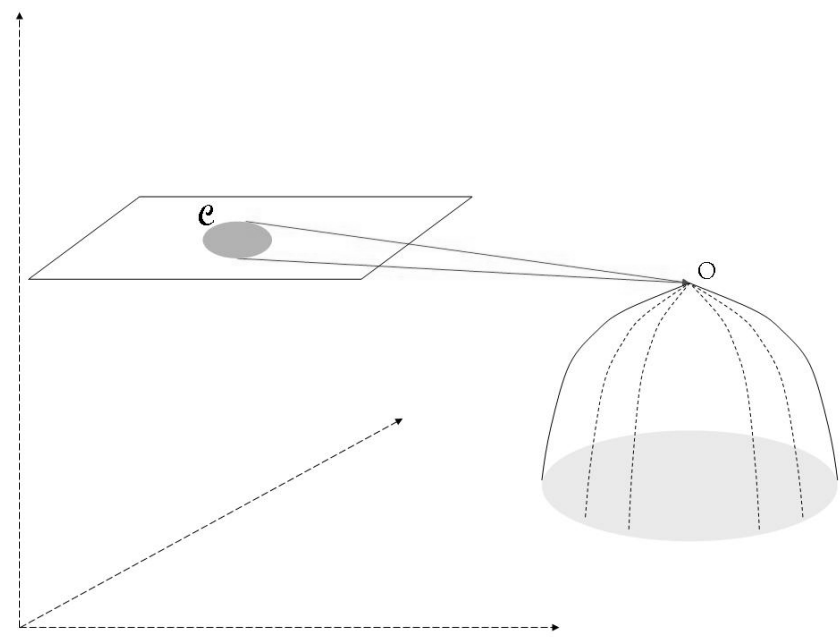

Fig. 2. The figure shows a defect obtained establishing a correspondence between a whole region of the reference manifold, $\mathcal{C}$, and one single point of the natural manifold, $O$. The defect induces a spontaneous strain in the natural manifold.

Vito Volterra ${ }^{13}$ in 1904 we may think a texture defect as corresponding to a missing or additional material which gives rise to a re-arrangement of the microscopic structure of the continuum. An example is visualized in Fig. 2.

In general a defect is obtained when a whole region of the reference manifold corresponds to a lower dimensional region of the natural manifold. In the figure a point-defect is represented, but it could as well have been a linear one.

Since we have in mind a physical process leading from the undeformed to the deformed manifold, the important feature of defects is that they induce a spontaneous strain field in the continuum, furthermore fixing the global symmetry.

In the case of four dimensions and a Lorentzian signature a complete classification of the possible defects is given in Ref. 14 .

\section{The Lagrangian Density of Space-time}

Insofar as the strain is considered as being the consequence of a real physical process and not simply a formal identification of pairs of points in two manifolds, it must be associated with what, in three dimensions, would be identified as a deformation or elastic potential energy. In the ordinary theory of elasticity strain is due to the presence of stresses originated either by external agents (forces on the boundary or in the bulk) or by the presence of defects. Between stresses, which are a tensor field $\sigma_{\mu \nu}$, and strain, in the linear theory, there is a simple linear relation which is Hooke's law:

$$
\sigma_{\mu \nu}=C_{\mu \nu}^{\alpha \beta} \varepsilon_{\alpha \beta}
$$


The rank 4 tensor $C_{\alpha \beta \mu \nu}$ is the elastic modulus tensor. When the medium is locally isotropic the elements of $C_{\alpha \beta \mu \nu}$ only depend on two independent parameters which are the Lamé coefficients of the material $\lambda$ and $\mu$. Under these considerations and assumptions the elastic energy density turns out to be:

$$
W=\frac{1}{2} \sigma_{\alpha \beta} \varepsilon^{\alpha \beta}=\frac{1}{2} \lambda \varepsilon^{2}+\mu \varepsilon_{\alpha \beta} \varepsilon^{\alpha \beta} .
$$

The scalar quantity $\varepsilon=\varepsilon_{\alpha}^{\alpha}$ is the trace of the strain tensor.

In practice $W$ is the unique second order scalar that can be built from the strain tensor and as such it should enter the Lagrangian density of space-time, since it represents a property of the manifold.

Summing up, we see that the present approach does not subvert General Relativity, simply introducing an additional term in the action integral. The new form of the action, including matter/energy, will then be:

$$
S=\int\left(R+\frac{1}{2} \lambda \varepsilon^{2}+\mu \varepsilon_{\alpha \beta} \varepsilon^{\alpha \beta}+\kappa \mathcal{L}_{\text {matter }}\right) \sqrt{-g} d^{4} x .
$$

All notations are standard; $\kappa=16 \pi G / c^{4}$ is the coupling constant of matter to geometry.

\section{A Homogeneous and Isotropic Universe}

We shall now use Eq. (6) to work out the properties of a Friedmann-LemaîtreRobertson-Walker (FLRW) universe. The homogeneity and isotropy of space strongly simplifies the equations. In fact the Robertson-Walker (RW) symmetry leaves just one free function in the line element, whose standard form is the well known:

$$
d s^{2}=d \tau^{2}-a^{2} d l^{2}
$$

According to our approach within the SST we may think that the global symmetry is induced by a localized defect, which is the SST version of the Big Bang. By the way, a defect, in the sense used here, is indeed a singularity, because there the curvature diverges. It is however a more manageable singularity than the one considered in the standard cosmology.

We directly consider a flat space, whose line element is $d l^{2}$, since this is the most commonly accepted hypothesis; the scale factor $a$ is a function of cosmic time $\tau(=c t)$ only. The strain tensor may easily be read out of the direct comparison of (7) with the flat Euclidean line element written using the same coordinates. In fact the choice of the correspondence between the coordinates on the reference and on the natural manifold is, in principle, not unique. In general, we may fix the coordinates of the reference introducing four free functions, as gauge functions, plus a constraint: to preserve flatness. Actually, if we want to use, for the reference manifold also, coordinates appropriate to a RW symmetry, only one gauge function 


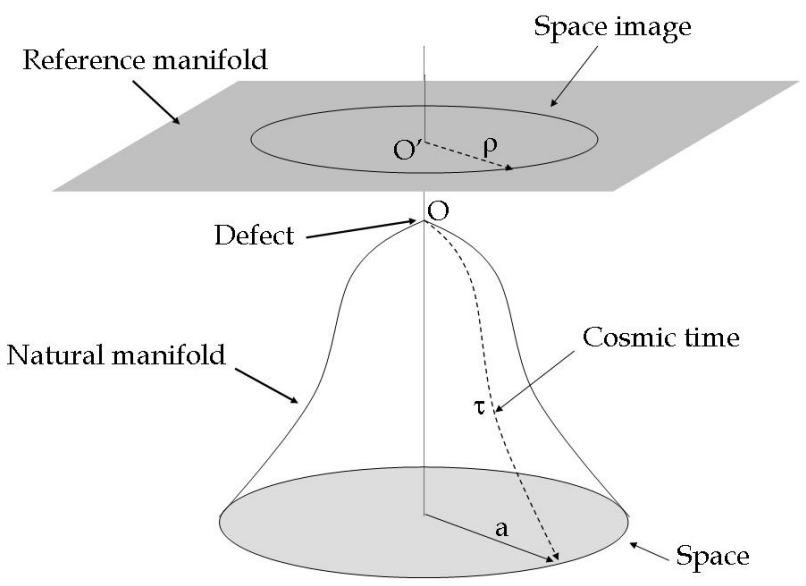

Fig. 3. A view of the embedding of a space-time endowed with a Robertson-Walker symmetry. The way the correspondence between the $\rho$ and the $\tau$ variable is established is expressed by the $b$ function (see text).

$b(\tau)$ remains and the Euclidean line element is written:

$$
d s_{E}^{2}=b^{2} d \tau^{2}-d l^{2}
$$

The situation is sketched in Fig. 3 .

From (7) and (8), using (2), we obtain the non-zero elements of the strain tensor (using Cartesian coordinates for space):

$$
\begin{aligned}
\varepsilon_{00} & =\frac{1-b^{2}}{2}, \\
\varepsilon_{i i} & =-\frac{1+a^{2}}{2} .
\end{aligned}
$$

The index $i$ runs from 1 to 3 and labels the space directions of a Cartesian frame.

Once the components of the strain tensor are defined we can fully exploit Eq. (6) deducing the equations for the two unknown functions $a$ and $b$. Finally, assuming that the matter is made of dust plus radiation, we get the evolution law for the scale factor in the form: ${ }^{10}$

$$
H=\frac{\dot{a}}{a}=c \sqrt{\frac{3}{16} B\left(1-\frac{(1+z)^{2}}{a_{0}^{2}}\right)^{2}+\frac{\kappa}{6}(1+z)^{3}\left[\rho_{m 0}+\rho_{r 0}(1+z)\right]}
$$

$H$ is the Hubble parameter; dot mean time derivative; $z$ is the redshift parameter; $a_{0}$ is the present value of the scale factor; $\rho_{m 0}$ and $\rho_{r 0}$ respectively are the present values of the mass and radiation densities in the universe; the Lamé coefficients are 
combined together in the $B$ parameter:

$$
B=\frac{3}{2} \mu \frac{2 \lambda+\mu}{\lambda+2 \mu} .
$$

Looking at (10) we see that the strain (represented by the term multiplying $B$ ) contributes a boost factor at any moment. For high values of $z$ (early times) radiation dominates and also the strain term assumes a radiation-like form, enhancing the expansion, which however decelerates. For small values of $z$ down to the limit $z=-1$ (positive infinity of time) the relevance of the radiation disappears and the effect of the $B$-term continues to be readable (in a $3+1$ splitting) as a push on the expansion rate producing a continuous asymptotically exponential acceleration.

\section{Cosmological Tests}

In order to put constraints on the values of the parameters of the theory, $B$ and $a_{0}$, as well as on $\rho_{m 0}$, we have used Eq. (10) for four typical cosmological tests. These are: the relative abundance of light isotopes from the Big Bang Nucleosynthesis (BBN); the acoustic horizon scale in the Cosmic Microwave Background; the Large Scale Structure (LSS) formation after the recombination era; the dependence of the luminosity of type Ia supernovae (SnIa) on the redshift. On all for cases the strain, influencing the expansion rate, can be expected to affect the observed results. The description and discussion of the tests is given in Ref. 11. Here we report the values of the parameters obtained from the analysis (it is $\left.B_{a_{0}}=\frac{8}{9} \kappa \rho_{r 0} a_{0}^{4}\right)$ ); the results and the related uncertainties are listed in Table 1.

Table 1. Optimal values of the parameters obtained from the four cosmological tests mentioned in the text.

\begin{tabular}{ccc}
\hline$\rho_{m 0}$ & $B$ & $B_{a 0}^{-1}$ \\
$\left(10^{-26} \mathrm{~kg} / \mathrm{m}^{3}\right)$ & $\left(10^{-52} \mathrm{~m}^{-2}\right)$ & $\left(10^{52} \mathrm{~m}^{2}\right)$ \\
\hline $0.260 \pm 0.009$ & $2.22 \pm 0.06$ & $0.011 \pm 0.006$ \\
\hline
\end{tabular}

The quality of the optimization is directly comparable with the results given by the standard $\Lambda$ Cold Dark Matter $(\Lambda \mathrm{CDM})$ model. The order of magnitude obtained for $B$ tells us the scale of the effects we may expect from the strain of space-time. Actually the influence of the "elasticity" of space-time appears at the scale of hundreds of Mpc.

\section{Conclusion}

We have illustrated how the formal correspondence between the properties and description of space-time in GR and the one of a material continuum may be given 
a physical interpretation. The curvature of space-time may be expressed in terms of strain of the manifold with respect to a flat and Euclidean reference. In this way the gravitational effects too are accounted for considering the difference between the given metric tensor and the metric tensor of the Euclidean frame. It is interesting to remark the role that texture defects have in the theory. They appear as localized discontinuities in the metric properties of the natural manifold and induce a spontaneous strained state. Defects define the specific symmetries of the problem, then the total curvature depends both on the defects and on the presence of matter/energy. By the way, the deformation process applied around a defect produces also the Lorentzian signature of space-time, discriminating the worldlines stemming out of the defect (timelike worldlines) from the others (spacelike lines).

This theory, when applied to the universe, produces interesting results. It leads to an accelerated expansion and it is possible to simultaneously and consistently deduce the optimal values of the parameters of the theory so that the data from observation of typical cosmic phenomena are reproduced with an accuracy comparable to the one obtained from $\Lambda$ CDM.

The Strained State paradigm seems promising but requires much further work. Other symmetries, besides the RW, are worth studying, such as the spherical symmetry in space (Schwarzschild symmetry) and the chiral symmetry, all thought as being due to a specific defect. Also propagating perturbations of space-time (gravitational waves) can be described in terms of specific solutions of the strain field, in which case they appear as "massive modes" with the troubles that these modes bring with them since the pioneering work of Fierz and Pauli. ${ }^{15}$

Even though all expected effects show up at the cosmic scale, it is legitimate to ask whether the theory is primary or it emerges from an underlying microscopic structure of space-time as it is the case of the classical three-dimensional elasticity. This question opens the door to the unsolved problem of the compatibility between quantum mechanics and the classical general relativistic approach of which SST is an extension.

All conclusions, after these remarks, are necessarily provisional, but the theory seems appealing and promising, having the advantage of being rooted in physical intuition and not only in mathematical consistency.

\section{References}

1. A. D. Sakharov, Sov. Phys. Dokl. 12, 1040 (1968).

2. H. Kleinert, Gauge Fields in Condensed Matter Theory, Vol II: Stress and Defects. (World Scientific, Singapore, 1989).

3. M. Pavšič, Grav. Cosmol. 1, 1 (1996).

4. S. S. Kokarev, Nuovo Cimento B 113, 1339 (1998).

5. C. Malyshev, Ann. Phys. 286, 249 (2000).

6. T. Padmanabhan, Int. J. Mod. Phys. D 13, 2293 (2004).

7. T. Padmanabhan, Braz. J. Phys. 35, 362 (2005).

8. A. Tartaglia, Gravit. Cosmol. 1, 335 (1995). 
9. A. Tartaglia, M. Capone and N. Radicella, The Cosmic defect theory tested by observation, in Proc. Int. Conf. on Problems of Practical Cosmology, eds. Y. V. Baryshev, I. N. Taganov and P. Teerikorpi (Russian Geographical Society, S. Petersburg, 2008), Vol. 2, p. 52.

10. A. Tartaglia and N. Radicella, Class. Quant. Grav. 27, 035001 (2010).

11. N. Radicella, M. Sereno and A. Tartaglia, Int. J. Mod. Phys. D 20, 1039 (2011).

12. J. D. Eshelby, Solid State Physics (Academic Press, New York, 1956).

13. V. Volterra, Ann. Sci. de lENS 24, 401 (1904).

14. R. A. Puntigam and H. H. Soleng, Class. Quantum Grav. 14, 1129 (1997).

15. M. Fierz and W. Pauli, Proc. R. Soc. A 173, 211 (1939). 